\title{
Equipment and technologies of air-plasma spraying of functional coatings
}

\author{
Viktor Kuzmin ${ }^{1,3,}$, Igor Gulyaev ${ }^{1}$, Dmitry Sergachev ${ }^{1}$, Sergey Vaschenko ${ }^{1}$, Elena Kornienko ${ }^{2}$, and Alexander Tokarev ${ }^{3}$ \\ ${ }^{1}$ Institute of Theoretical and Applied Mechanics SB RAS, Novosibirsk, Russia \\ ${ }^{2}$ Novosibirsky State Technical University, Novosibirsk, Russia \\ ${ }^{3}$ Siberian State University of Water Transport, Novosibirsk, Russia
}

\begin{abstract}
This article presents a short description of the DC plasma torch "PNK-50" structural features (ITAM SB RAS, Novosibirsk) used for spraying of functional coatings with powder materials as well as gives the results of thermophysical and technological studies of spraying regimes. We present preliminary results of the plasma torch supersonic modification development and the results of industrial approbation of the plasma torch and of multi-purposes functional coatings deposition processes.
\end{abstract}

\section{Introduction}

Plasma spraying is one of the effective methods of $0.1 \mathrm{~mm}$ and more thickness functional coatings applying. Practically any powder materials of $20 \ldots 100 \mu \mathrm{m}$ dimension (ceramic, metal, cermet, etc.) can be sprayed with the help of thermal plasma fluxes. Atmospheric plasma spraying by using air as a working gas (airplasma spraying) deserves particular attention. This technology allows reducing the cost and payback period of equipment. Moreover, the plasma spraying allows realization of plasma jet temperature and dynamic characteristics which ensure the necessary conditions for the deposition of high-quality coatings of various categories.

The present article demonstrates the possibilities of modern air-plasma plant on the example of the plasma torch "PNK-50" and considers the approach to the development and optimization of deposition technological regimes using diagnostic equipment and examples of industrial approbation of technology.

\section{The plasma torch for powder spraying}

The plasma torch "PNK-50" is constructed according to a linear scheme with a sectioned interelectrode insert (IEI) [1]. It is designed for operation in turbulent (highspeed), transient and laminar (low-speed) regimes of plasma jets outflow. It allows high-efficiency deposition of both metallic and ceramic powders (Fig. 1, a). The gas-discharge chamber of the plasma torch is a channel widening from the cathode to the anode. It consists of the sections of IEI, which are electrically isolated from each other and from the electrodes (Fig. 1, b).

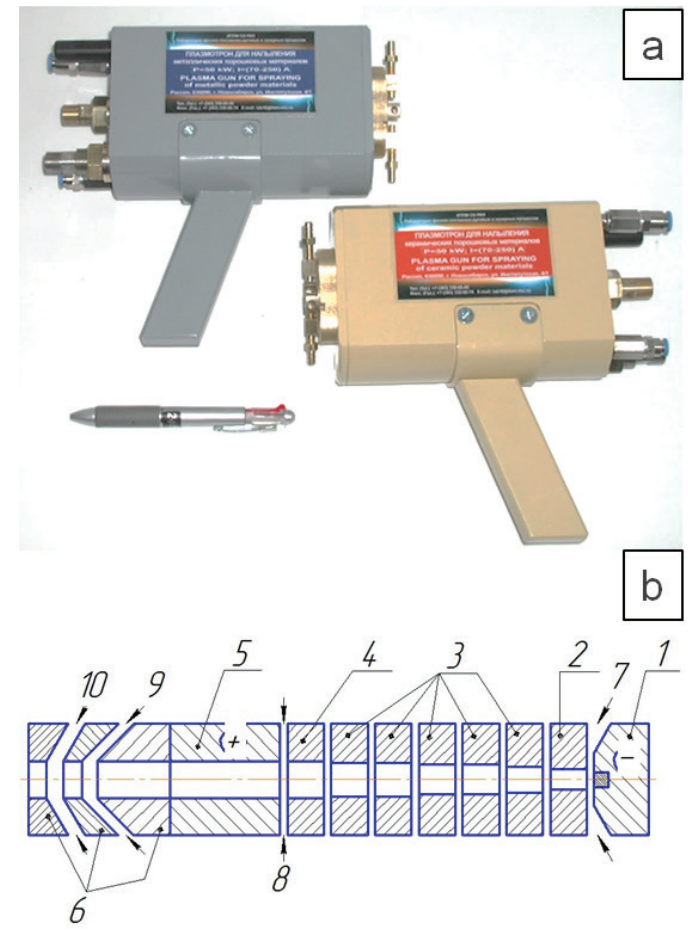

Fig. 1. Plasma torch «PNK-50»:

a - appearance of plasma torch version for manual operation; $\mathrm{b}$ - plasma torch channel scheme:

1 - cathode; 2 - starting insert; 3 - IEI inserts; 4 -transition insert; 5 - anode; 6 - unit for annular injection of powder with gas-dynamic focusing; 7 - plasma-forming gas; 8 - shielding gas; 9 - powder with transporting gas; 10 - focusing gas.

The use of IEI with a different number of inserts (from 1 to 5) allows changing the average arc length and, consequently, the arc voltage and the power of the plasma torch for the implementation of different deposition regimes: "ceramics", "metal", "supersonic". In addition, the scheme of channel with IEI permits to repress unwanted pulsations of length and arc voltage.

\footnotetext{
* Corresponding author: vikuzmin57@mail.ru
} 
The key element of the plasma torch construction is the original unit for annular injection of powder with gas-dynamic focusing (Fig. 1, b) [2]. This injection scheme ensures the material input in the axial part of the plasma flow, which is characterized by the higher values of speed and temperature. As a result, the efficiency of heating, acceleration of the particles and the deposition productivity are increased.

Air is used as the main working gas for plasmaforming, shielding, transporting and focusing. Small amount of combustible gas (propane-butane or methane) is added to the air which is used as protection (screen) of the plasma torch anode. If necessary, the combustible gas is added to the air which is used as a transporting and focusing gas. Air constitutes a high-enthalpy working gas being at the same time the most accessible and cheap one. The addition of combustible gas prevents the oxidation of the plasma torch anode working surface and the sprayed metallic powders.

\section{Thermophysical studies and coatings deposition}

The development and control of deposition regimes of coatings with specified functional properties requires a careful analysis of the sprayed material particles characteristics in the jet. Primarily, it is their speed and temperature at the deposition distance. In this work, we used the original system of express control. This system was developed by Yugra State University and ITAM SB RAS [3-5]. It allows scanning of a sprayed jet to measure velocity and temperature of the sprayed particles (Fig. 2).
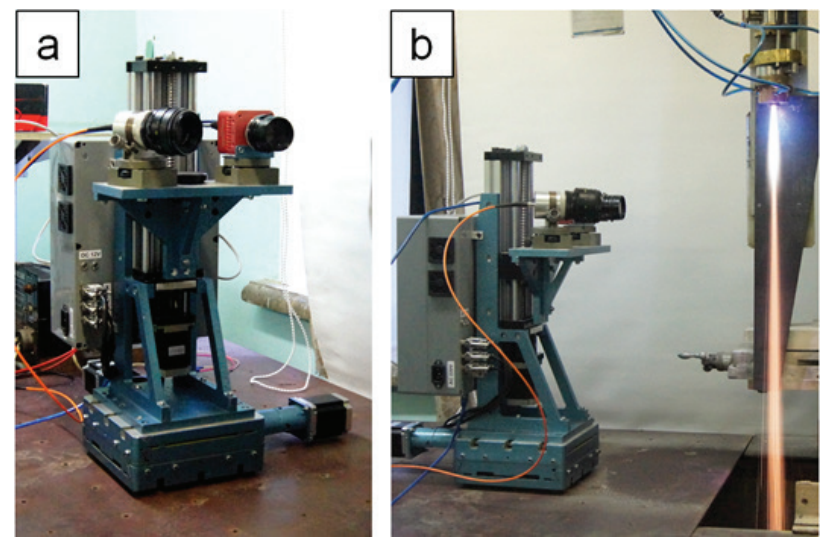

Fig. 2. Optical system for diagnostics of dispersed phase of spraying jet: a - appearance of diagnostics mounted on 3Dpositioning system; $b$ - analysis of self-fluxing $\mathrm{NiCrBSiC}$ powder spraying regime.

Fig. 3 presents the change graphs of the average values of the particles velocity and temperature along the jet length for different arc currents for spraying of selffluxing powder (NiCrBSiC system) in order to demonstrate the spraying modes optimization technique. The data suggest that in order to achieve maximum velocity $(270 \ldots 290 \mathrm{~m} / \mathrm{s})$ and temperature $(2400 \mathrm{~K})$ of particles on this system, the deposition should be made at an arc current of at least $140 \mathrm{~A}$ and the spraying distance of maximum $200 \mathrm{~mm}$.
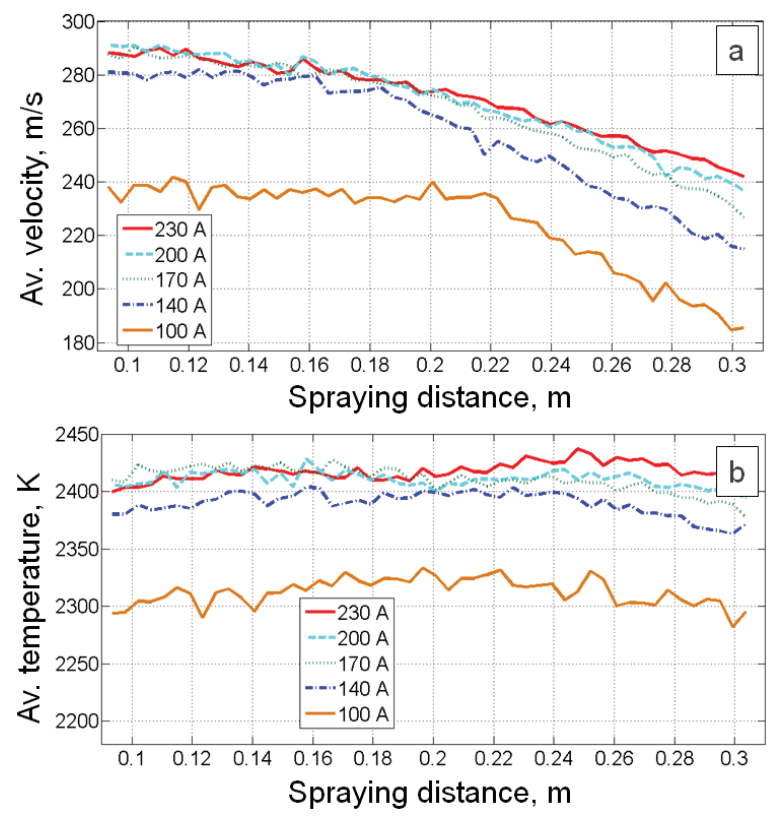

Fig. 3. Dependence of average particle velocity (a) and temperature (b) vs spraying distance for various electric arc currents. NiCrBSi powder $40 \ldots 100 \mathrm{um}$. Plasma torch configuration «Metal».

The obtained data about the particles parameters in the sprayed jet were used to optimize the spraying regimes of the NiCrBSiC material. This material is used as wear-resistant, corrosion-resistant and heat-resistant coatings. Coatings spraying at the arc current of $140 \mathrm{~A}$ and at the distance of $170 \mathrm{~mm}$ are characterized by the absence of unmelted particles. Their porosity is of less than $1 \%$ with more than $50 \%$ of the pores having size smaller than $5 \mu \mathrm{m}$. The transmission electron microscopy method revealed that microcrystalline structure with grains average size of $80 \mathrm{~nm}$ was formed during deformation and quench of the material particles $[6,7]$. This effect leads to the increase of the coatings hardness in comparison with the hardness of the original powder (more than $20 \mathrm{HRC}$ ).

\section{Development of the supersonic plasma torch modification}

The deposition of dense coatings from metal and carbide (WC/Co, $\mathrm{WC} / \mathrm{Ni}$ ) materials requires a high particle velocity $(500 \ldots 800 \mathrm{~m} / \mathrm{s}$ and higher). For that, we calculated the geometry of the unit for annular injection of powder in the form of a supersonic Laval nozzle. The nozzle diameter in the critical section was $6 \mathrm{~mm}$ and the Mach number at the nozzle cut was 1.5. Integral characteristics of the plasma torch at supersonic regimes in correlation with the current strength of arc discharge are presented in Table 1.

The use of the optical system for diagnostics allows investigation of the spatial structure of a plasma jet, as well as measuring the velocity and temperature of a dispersed phase in the process. Fig. 4, a presents the jet 
during the deposition of Ni-Cr-Si-B-C wear-resistant material with $20 \ldots 63 \mu \mathrm{m}$ dimension.

Table 1. Properties of supersonic air plasma jet at various electric arc currents (I): arc voltage (U), thermal power (Q), static pressure $(\mathrm{P})$, mean plasma temperature $(\mathrm{T})$, mean plasma velocity (V).

\begin{tabular}{|c|c|c|c|c|c|}
\hline $\mathbf{I ,} \mathbf{A}$ & $\mathbf{U}, \mathbf{B}$ & $\mathbf{Q}, \mathbf{K B} \mathbf{T}$ & $\mathbf{P ,} \mathbf{a T a}$ & $\mathbf{T ,} \mathbf{K}$ & $\mathbf{V , \mathbf { M } / \mathbf { c }}$ \\
\hline 110 & 285 & 22,1 & 0.79 & 2737 & 1484 \\
\hline 140 & 278 & 26,5 & 0.85 & 3039 & 1564 \\
\hline 180 & 268 & 32,5 & 0.92 & 3330 & 1642 \\
\hline 220 & 262 & 38,1 & 0.96 & 3574 & 1737 \\
\hline
\end{tabular}

The particles flux is seen to have a dense structure with angle divergence of not more than $5^{\circ}$. Fig. $4, \mathrm{~b}$ contains the image of the jet initial section (without powder) in which the system of compression rises (Mach's disks) of a slightly overexpanded supersonic flux is clearly seen. The blue color at the jet periphery corresponds to the protective envelope of propanebutane which is added to the transporting and focusing air to reduce the oxidation of the sprayed particles. The structure visualization of the supersonic jet obtained by mathematical processing of the monochrome image of the jet is shown in Fig. 4, $\mathrm{c}$ and d.
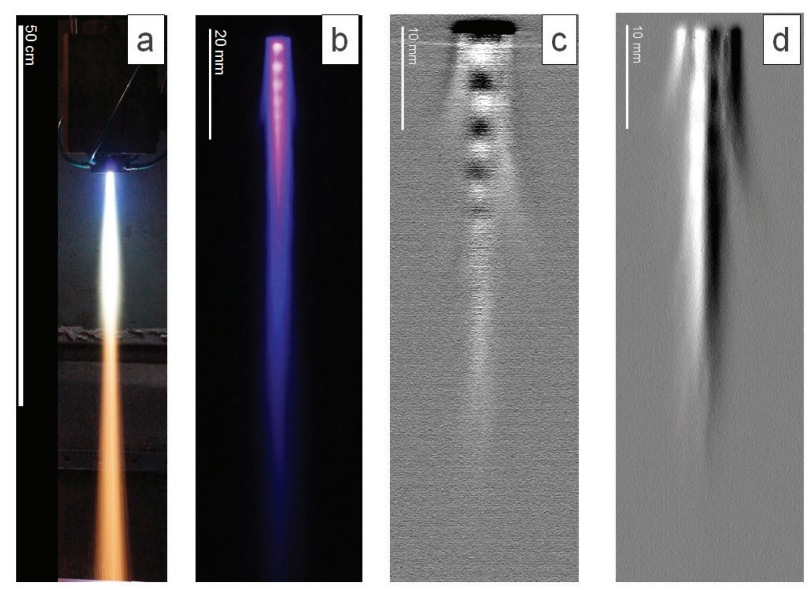

Fig. 4. Supersonic air-plasma jet: a - spraying process of NiCrBSiC powder, $\mathrm{b}$ - supersonic air plasma jet with shielding envelope of propane/butane, $\mathrm{c}$ - visualization of axial structure of jet - shock diamonds (Mach's discs), d - visualization of radial structure of jet - main central jet and shielding peripheral envelope.

The diagnostic of the sprayed jet showed that the use of a supersonic spraying regime allows significant increase in the average particle velocity at the spraying distance from 300 to $500 \mathrm{~m} / \mathrm{s}$. The stream of particles in the supersonic jet is more concentrated: the width of the jet is $9.6 \mathrm{~mm}$ compared to $14.7 \mathrm{~mm}$ in a subsonic jet. It is known that a concentrated sprayed jet provides more uniform values of the particles velocity and temperature in the sprayed spot.

This increase in the sprayed particles velocity in the supersonic regime allows achieving the values of the coatings microhardness of $693 \mathrm{HV}$ that is $20 \ldots 25 \%$ higher in comparison with the subsonic regime (Table 2). The hardness increase can be explained by the formation of a substantially finer-grained structure of coatings in the case of supersonic spraying, as the closing time of the crystallization front of a liquid particle with a free surface at the impact against a substrate is inversely proportional to its velocity immediately before the impact. Accordingly, the higher is the velocity of the sprayed particles, the shorter is the time of their crystallization during the spreading on the substrate.

Table 2. Spraying modes oomparison of $\mathrm{NiCrSiB}$ powder with size $20 \ldots 63 \mathrm{um}$

\begin{tabular}{|l|c|c|}
\hline \multicolumn{1}{|c|}{ Spraying mode } & Subsonid & Supersonic \\
\hline $\begin{array}{l}\text { Plasma torch electrical power, } \\
\mathrm{kW}\end{array}$ & 48 & 52 \\
\hline \multicolumn{3}{|c|}{ Plasma jet characteristics } \\
\hline Plasma velocity, m/s & 1200 & 1650 \\
\hline Plasma temperature, $\mathrm{K}$ & 3800 & 3200 \\
\hline \multicolumn{3}{|c|}{ Powders flow characteristics on spraying distance } \\
\hline Spraying distance, mm & 170 & 200 \\
\hline Powders velocity, m/s & $307 \pm 62$ & $504 \pm 68$ \\
\hline Powders temperature, K & $2845 \pm 14$ & $2938 \pm 110$ \\
\hline Jet diameter, mm & 5 & 9.6 \\
\hline \multicolumn{3}{|c|}{ Coatings characteristics } \\
\hline Overall porosity, \% & 3 & 5 \\
\hline Average size of pores, $\mu \mathrm{m}$ & 13 & 10 \\
\hline Microhardness, HV & $558 \pm 61$ & $683 \pm 108$ \\
\hline
\end{tabular}

\section{Industrial approbation of air-plasma spraying technology}

The industrial plant of plasma spraying "Thermoplasma 50-01" was developed and manufactured on the basis of the plasma torch "PNK-50". It includes a power source, a control panel, a switching module with a start-up unit, two powder dozers and an unit of autonomous cooling of plasma torch (Fig. 5). The plant is equipped with two plasma torches. They can be used both in manual and mechanized versions. The channel of one of the plasma torches is assembled for the spraying of metallic powders, the channel of the other plasma torch was assembled for the spraying of ceramic powders. The switching of the power supply, cooling, gas supply, the unit for starting and feeding of the powder from one plasma torch to the other is done remotely from the control panel. 


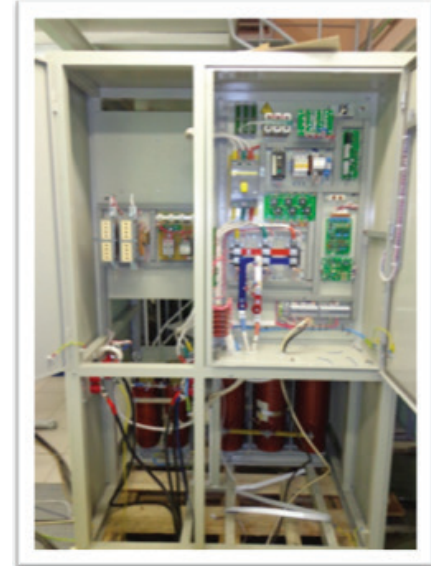

Power source

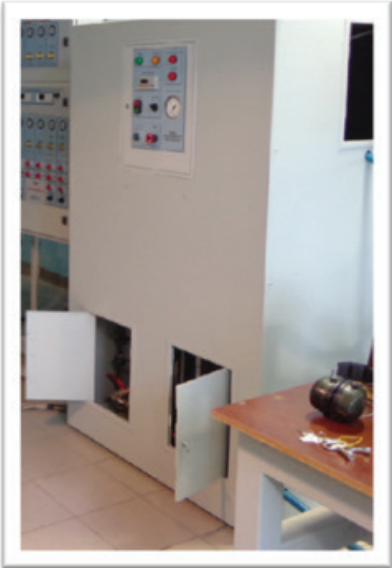

Chiller

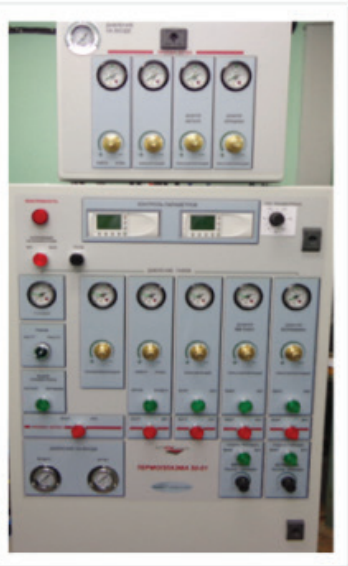

Control panel
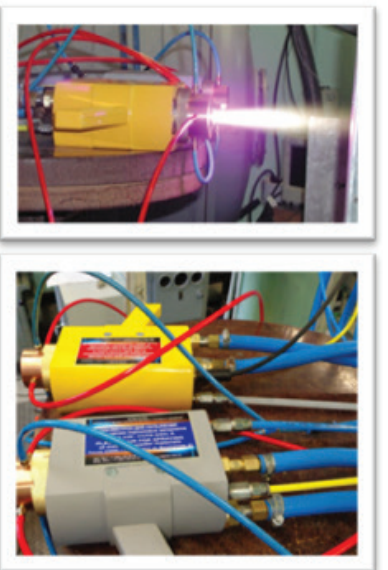

Plasma torches
Fig. 5. Basic components of spraying facility «Thermoplasma 50-01».

Fig. 6 presents the photographs of a number of parts with various functional coatings deposited with the help of the plasma torch "PNK-50".

The high quality of the coatings obtained with the plasma torch "PNK-50" allowed to create a new technology for protecting of the details of the ships from hydroabrasive and cavitation wear. In this case a coatings flowing operation after the spraying is not required. The screw propeller of the small ship "RC101D" with wear-resistant coatings looks outwardly practically not worn-out after the navigation in 2016 in hard conditions of the shallow waters of the Biya and Katun rivers (Fig. 7, a). This screw is suitable for exploitation in the next navigation period. The control screw propeller (without the protective coating) requires renewal during navigation (welding plates to worn edges and surfacing of the worn surfaces (Fig. 7, b).
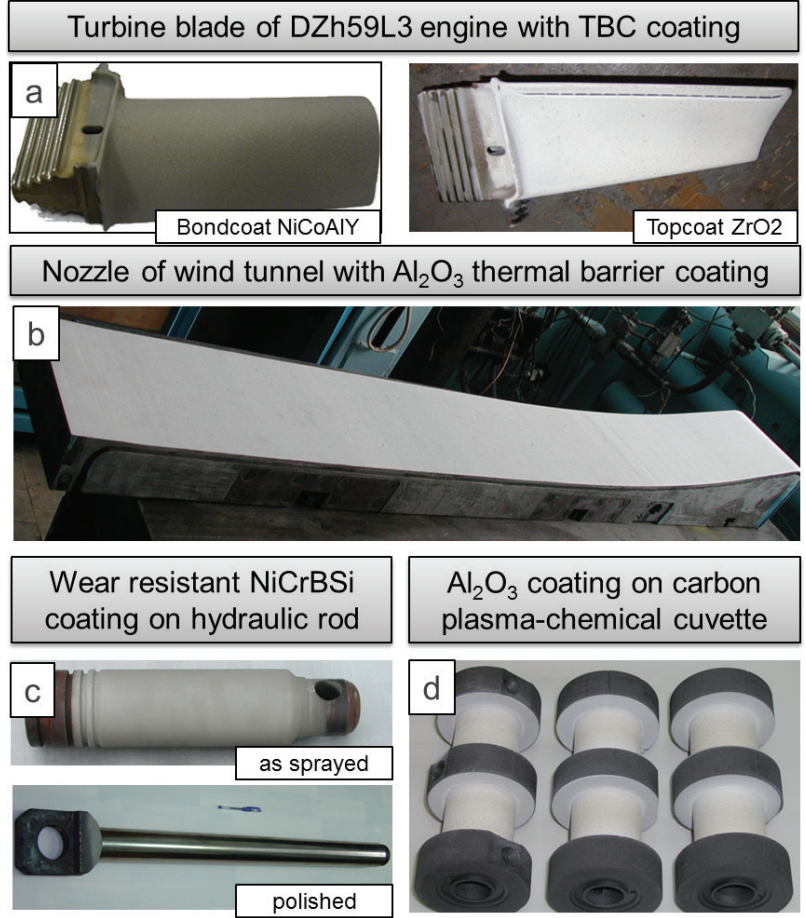

Fig. 6. Functional coatings produced by air-plasma spraying: a -DZh59L3 engine high pressure turbine blade with internal cooling restored with double-layer thermal barrier coating; $b$ nozzle element of wind tunnel with hydrogen heater. Coated surface $1300 \times 300 \mathrm{~mm}$, coating thickness $500 \mathrm{um}$; c - rod of hydraulic cylinder manipulating powered supports for mining facilities; d - carbon cuvettes for spectroscopic analysis of high-temperature decomposition in plasma-chemical reactor.
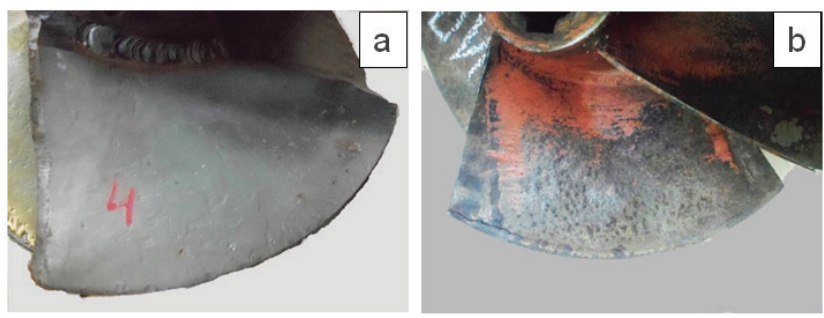

Fig. 7. Blades of project «KS-101D» boat propeller after navigation in 2016 season: a - blade with protective coating $\mathrm{NiAl} ; \mathrm{b}$ - blade without coating, required restoration during navigation period.

\section{Conclusion}

Air-plasma spraying of coatings is one of the most universal methods of gas-thermal spraying. It combines a wide range of the applied materials (from metals to ceramics and composites), high productivity and low cost of technology. Systematized studies of electric arc processes, interphase interaction in high-temperature dispersed streams, and coatings formation processes allowed developing of a new generation air plasmatron "PNK-50", as well as spraying technologies which have been introduced into production. 


\section{References}

1. V.I. Kuzmin, A.A. Mikhalchenko, V.I.Nalivaiko, P.A. Chubakov, Russian Physics J. 50, 85-88 (2007).

2. V.I. Kuzmin, A.A. Mikhal'chenko, O.B. Kovalev, E.V. Kartaev, N.A. Rudenskaya, Journal of Thermal Spray Technology. 21,159-168 (2012)

3. A.V. Dolmatov, I. P. Gulyaev, V.I. Jordan, IOP Conference Series: Materials Science and Engineering 81, 012041 (2015).

4. A.V. Dolmatov, I.P. Gulyaev, P.Yu. Gulyaev, V.I. Jordan, IOP Conf. Series: Materials Science and Engineering 110, 012058 (2016).

5. 5. A.V. Dolmatov, I.P. Gulyaev, V.I. Kuzmin, E.A. Lyskov, K.A. Ermakov, Thermophysics and Aeromechanics 24, 83-94 (2017).

6. E.E. Kornienko, E.J. Lapushkina, S.P. Vaschenko, I.P. Gulyaev, E.V. Kartaev, D.V. Sergachev, N. Kashapov, S. Sharifullin, I. Fayrushin, Journal of Physics: Conference Series 567, 012010 (2014).

7. E. E. Kornienko, D. O. Mul', O. A. Rubtsova, S. P. Vaschenko, V. I. Kuzmin, I. P. Gulyaev, D. V. Sergachev. Thermophysics and Aeromechanics 23, 919-927 (2016) 